\title{
Research on Open Source Software Intended to Promote its Usage in Education
}

\author{
Valentina Dagiene \\ Institute of Mathematics and Informatics, Akademijos str. 4, \\ LT-08663 Vilnius, Lithuania dagiene@ktl.mii.lt \\ WWW home page: http://ims.mii.lt/ims/asmen/valentina/
}

\begin{abstract}
The paper deals with the use of open source software in education. Open source software is used more and more all over the world. Linux, Mozilla, MySQL, OpenOffice.org etc. are very well-known packages. A lot of open source programs are developed especially for educational purposes; virtual learning environments, editors, simulators, microworlds, subject-based learning applications, etc. The main problem of this field is to involve educators and policy makers to disseminate effectively information about open source, and to bring students and teachers together for improving open code. The investigations on open source are being fulfilled in a few Lithuanian institutions. The main objectives are to validate the economic and pedagogical utility of open source software in schools.
\end{abstract}

\section{Introduction}

The vision of the open source community is to produce better software than the traditional closed model, in which only a very few programmers can see the source and everybody else must blindly use an opaque block of bits. "The basic idea behind open source is very simple: When programmers can read, redistribute, and modify the source code for a piece of software, the software evolves. People improve it, people adapt it, people fix bugs. And this can happen at a speed that, if one is used to the slow pace of conventional software development, seems astonishing" is declared in an open source initiative site [8]. 
The main feature of open source software lies in the possibility to use the code of program applications. Besides this open source provides the following possibilities [1]: a) to use the software in a desirable way, b) to adapt the software to one's needs, to explore it, to correct the detected bugs, and to improve its functionality, and c) to distribute the software $[5,6]$.

Open source software is very important in education: 1) the license is free and can be used in all institutions without restrictions, 2) the license does not restrict anyone from making use of the program in a specific field of endeavour, 3) applications may be improved, adapted, localized, etc. faster and easier, generally without long negotiation procedures, and 4) open source is real stuff for investigation and analyses of code for novice programmers and everyone else who wants to improve in building software.

The problem of the use of illegal software and piracy is experienced to a varying degree by all countries in the world. Countries try to find different solutions to the problem according to their financial capabilities, cultural and intellectual awareness of people, and traditions and methods of dissemination of information [3]. Using open source software is one of the most straightforward and effective methods to combat the use of illegal software.

Open source software not only helps in tackling the problem of illegal use of software but also has a more powerful mission: it allows the modification of program code, quick correction of detected bugs and uniting the efforts of a number of programmers.

Open source software may be quickly localised without any negotiation obstacles. However the degree of open source software internationalization is lower than that of commercial software, and the translations of a lot of open source software that is published on the internet are incomplete and, sometimes, just in the initial stage of translation $[4,7]$.

\section{The Lithuanian study on open source}

Lithuanian state policy has a quite positive approach to open source software: various workshops and seminars are being held, appropriate resolutions that recommend implementation of open documents (based on open standards) are being adopted, and different initiatives on open source are being sponsored. A study on open source [9] was done to evaluate how the ideas of open source reach schools and how teachers and pupils use open source software in practice. The scope of the study allowed focusing on more specific research, i.e., the analysis of open source software used in education.

The goal of the study Open Source in Education was to analyse the situation of open source software in Lithuania as well as in other European countries: how open source software is used in education, what are the main projects and initiatives, and how open source applications are adapted, localised and supported.

Basically the study of open source software was carried out in two directions: 1) analysis of articles, documentation and collection of different information related to 
open source software in education; and 2) a questionnaire survey was conducted in Lithuanian schools and the results were analysed, systemised and described.

The survey of schools regarding open source software was based on the research approach with the entire predefined model of work and empirical research [2].

The following objectives were formulated in order to reach the goal: 1) to analyse scientific, methodological and information sources on open source software and to discuss their theoretical aspects, 2) to investigate the methods of development, localisation and distribution in the European Union and other countries, 3) to analyse the development of open source software in educational systems of various countries, 4) to carry out the survey (questionnaire survey) in Lithuanian secondary schools regarding open source software, and 5) to describe the economic and educational benefits of open source software and to develop recommendations.

The population chosen for the study was composed of all secondary schools (511) and gymnasia (92) in Lithuania. A simple random sample of 23 gymnasia and 31 secondary schools was selected for the survey. The population studied in the students' research was composed of all twelfth grade students of these schools. The two-level cluster sample plan was used: the systematic sample plan was used individually in every twelfth grade class where every fifth student was selected from the class-list starting with the second student on the list.

\section{Open source software in secondary schools}

On average Lithuanian comprehensive schools and gymnasia have 24 computers each. Results of the study imply that the best situation regarding the number of available computers was in gymnasia (approx. 35 computers each) and the worst one was in rural secondary schools (approx. 20 computers each). All schools have access to the internet, and $41 \%$ of Lithuanian schools have their own servers. Nearly $40 \%$ of these schools use the Linux operating system.

One pack of OpenOffice.org (containing a $C D$ with the documentation) was appropriated for each school and provided for each Board of Education. However the OpenOffice.org suite was installed in approximately 2 school computers.

The survey has shown that even $29 \%$ of secondary schools in cities introduce just the Microsoft Office packs, although both the curricula and the textbooks constantly refer to open source applications OpenOffice.org and Mozilla as well. A total of $61 \%$ of all schools just briefly discuss the open source programs and use only the Microsoft applications (Figure 1) (Secondary C - Secondary schools in cities, Secondary D - Secondary schools in districts).

The choice of open source applications is mostly prompted by the need to introduce alternative software to students; this answer was chosen by $69 \%$ of school teachers. The other important reason for the use of open source software lies upon the fact that it is free of charge; consequently schools may save money on licenses $(56 \%)$. The other reasons were less important.

Even $85 \%$ of teachers think that the spread of open source software is considerably insufficient. As the main reason for this, teachers suggest the lack of literature on working with open source software (this answer was chosen by $52 \%$ of 
teachers), the lack of information on open source software (37\%), the conviction that some problems may occur during exchange of data with Microsoft users (40\%), and the lack of time to practice open source software (39\%).

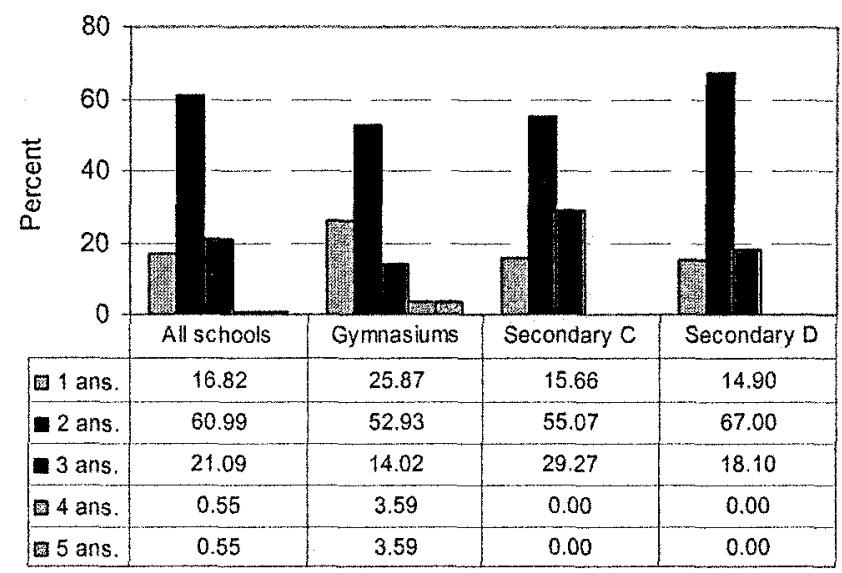

Figure 1. Do you introduce both commercial and open source software to students? Yes, we introduce both types of the software ( $I$ ans). Partially yes: we briefly discuss open source, though we use just Microsoft software (2 ans). No, we introduce just Microsoft software (3 ans). No, we introduce just open source software (4 ans). Other ( 5 ans $)$.

In 2005-2006 the optional programming exam based on using the Free Pascal environment in secondary schools is scheduled. At the moment Turbo Pascal or Borland Pascal is being often used in schools. The research indicates that in schools that employ advanced teachers of information technology and computer specialistsengineers the FPK compiler is widely used (67\%), while schools in cities and districts use the FPK compiler much less, respectively $33 \%$ and $28 \%$.

According to the answers it may be assumed that the issues of software legality are being quite widely discussed in schools. Besides this, it appears that schools also quite fairly know the organizations promoting open source programs; $65 \%$ of all respondents do believe that the open source movement will develop in the future.

Schools are much more interested in stable and properly localized open source programs than in every localization version that may be of lower quality. Additionally, schools are very interested in printed manuals for software.

\section{Students attitudes towards open source software}

According to the study, $44 \%$ of all students have never heard about open source software, although their textbooks contain appropriate information. Most students become aware of open source programs from their friends (31\%) and teachers (27\%) and $9 \%$ have found open source software on the internet. According to students $(70 \%)$, the most attractive feature of such applications is that they are free of charge 
and may be legally used. Other features were less attractive: possibility to improve them (18\%), user-friendly environment (21\%), and better virus resistance $(12 \%)$.

Students, like teachers, would prefer stable and localized open source software $(77 \%)$. Differently from teachers, students are equally friendly to both types of open source software's dissemination: via the internet (38\%) and with printed description $(32 \%)$. Nevertheless students from gymnasia prefer the internet $(51 \%)$. Almost all students are equally interested in localization of open source software.

Most students $(67 \%)$ have no open source software on their computers. Just the OpenOffice.org suite is somewhat more interesting to them (19\%), but $43 \%$ have friends and acquaintances who use open source software. Most (64\%) stated that open source programs are used too little during the classes because of the lack of appropriate information; $50 \%$ of the students responded that they get acquainted just with Microsoft office suite during the classes (Figure 2).

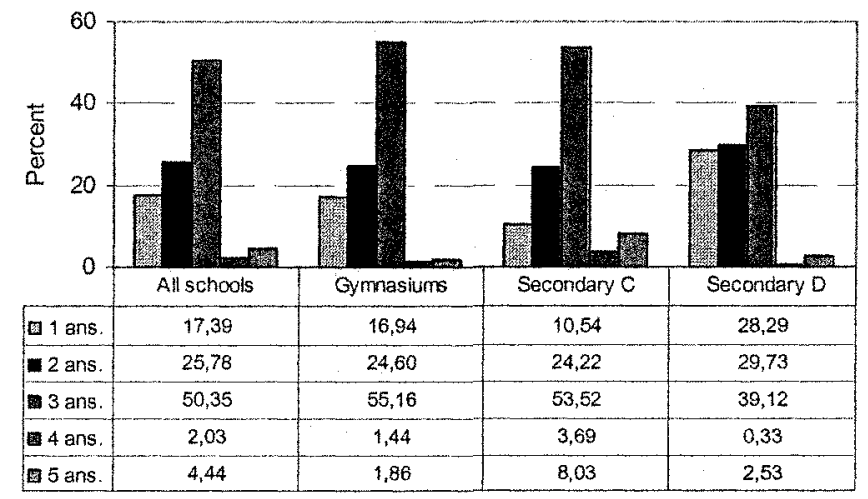

Figure 2. Do you get acquainted both Microsoft and open source programs OpenOffice.org and Mozilla during the classes? Yes, teacher introduces both types of software (1 ans). Partially yes: we briefly discuss open source, though we use just Microsoft software (2 ans). No, we get acquainted just with Microsoft software ( 3 ans), No, we get acquainted just with open source software (4 ans). Other (5 ans).

\section{Conclusions}

Considering the situation related to the use of open source software, and bearing in mind the results of the study on matters related to open source software in schools of Lithuania, the guidelines for improving the existing situation were elaborated:

(a) The development of open source software in the country reduces the prices of commercial software and gives impetus for the translation of software into the local language. It is necessary to take care of cultural and linguistic quality of open source software, and this matter should not be entirely left to the enthusiasts.

(b) The promotion and support for open source software should help solve the problem of the legality of software. Since open source software does not have to be bought, the funds saved can be better invested into the improvement, adaptation and maintenance of open source software. 
(c) Officially drawn up documents or other information must be available both for users of commercial and open source software so that the users of open source software have the same opportunities as others. Documents and internet services must be provided according to open source formats.

(d) As many as 20 different versions of Linux that are adapted to teaching have been developed in various countries, and they are used in individual countries or their groups. It should be required to analyse them and determine which one would be appropriate to be localised and used in schools.

(f) Students should be introduced to general purpose software of both types: commercial software necessary for teaching and learning as well as to equivalent open source software. When examinations on information technologies are prepared for general education schools, it is necessary to provide the possibility to use general purpose software of both types: commercial software and open source software.

(g) To ensure that schools receive open source software of good quality, it is necessary to implement quality assurance measures (testing, reviewing in terms of functionality, language, design, etc.) at all stages of software development from selection thorough to testing of localised software.

\section{References}

1. A Brief History of Free/Open Source Software Movement (2006): http://www.openknowledge.org/writing/open-source/scb/brief-open-source-history.html

2. Anderson, G., Arsenault, N.: Fundamentals of Educational Research. RoutledgeFalmer, Taylor \& Francis Inc. (1998)

3. Dagiene, V., Hammond, M., Hardarson, A., Wazlawick, R. Weber, W.: Social, Ethical and Cognitive Issues of Informatics and ICT. In: Network the learner: computers in education: Seventh IFIP World Conference on Computers in Education, WCCE 2001, July29-August 3, 2001, Copenhagen. D. Watson, J. Andersen, (Eds.), Kluwer Pub. (2002) 853-859

4. Dagiene, V., Laucius, R.: Internationalization of open source software: framework and some issues. In: 2nd International Conference Information Technology: Research and Education, T. Boyle, P. Oriogun, A. Pakstas (Eds.), 28 June - 1 July, London (2004) 204207

5. European Commission: Open Source Observatory. Case Studies zooming in on certain Open Source adoptions in Member States. http://europa.eu.int/idabc/en/chapter/470 (2005)

6. Hopfner, H.: Open Source Software in Education: A Report of Experience. Informatics in Education, vol. 2, no. 1 (2003) 15-20

7. Jevsikova, J., Dagiene, V., Grigas,: G. Mozilla internet application suite: developing for education. In: 2nd International Conference Information Technology: Research and Education, T. Boyle, P. Oriogun, A. Pakstas (Eds.), 28 June-1 July, London (2004) 96100.

8. Open Source Initiative: http://www.opensource.org/index.php (2006).

9. Report on study of open source in education: Ministry of Education and Science of Republic of Lithuania. http://www.ipc.lt/21z/duomenys/tyrimai/atviras\%20kodas/ataskaita.pdf (2004). 